\title{
Efficient Resource Management Mechanism with Fault Tolerant Model for Computational Grids
}

\author{
R. Kohila \\ Department of Computer Science and Engineering \\ V.S.B Engineering College \\ Tamilnadu, India.
}

\begin{abstract}
Grid computing provides a framework and deployment environment that enables resource sharing, accessing, aggregation and management. It allows resource and coordinated use of various resources in dynamic, distributed virtual organization. The grid scheduling is responsible for resource discovery, resource selection and job assignment over a decentralized heterogeneous system. In the existing system, primary-backup approach is used for fault tolerance in a single environment. In this approach, each task has a primary copy and backup copy on two different processors. For dependent tasks, precedence constraint among tasks must be considered when scheduling backup copies and overloading backups. Then, two algorithms have been developed to schedule backups of dependent and independent tasks. The proposed work is to manage the resource failures in grid job scheduling. In this method, data source and resource are integrated from different geographical environment. Faulttolerant scheduling with primary backup approach is used to handle job failures in grid environment. Impact of communication protocols is considered. Communication protocols such as Transmission Control Protocol (TCP), User Datagram Protocol (UDP) which are used to distribute the message of each task to grid resources.
\end{abstract}

Key Words: Grid Computing, Primary Backup, Communication Protocols, TCPTransmission Control Protocol, UDP- User Datagram Protocol.

\section{INTRODUCTION}

\subsection{Grid Computing}

Grid Computing is distributed; large scale cluster grid computing has emerged as the next-generation parallel and distributed computing methodology, which aggregates dispersed heterogeneous resources for solving various kinds of large-scale parallel applications in science, engineering and commerce. It can integrate and utilize heterogeneous computational resources from different networks or regional areas into a high performance computational platform and can solve complex computing-intensive problems efficiently. Grid service represents convergence between high performance computation and web service. Grid aims ultimately to turn the global network of computers into a vast computational resource.

\subsection{Grid Computing Overview}

A distributed heterogeneous computing system consists of a distributed suite of different high-performance machines, interconnected by the high-speed networks, to perform different computationally intensive applications that have various computational requirements. Heterogeneous computing systems range from diverse elements or paradigms within a single computer to a cluster of different types of personal computers to coordinate geographically distributed machines with different architectures. Job scheduling is one the major difficult tasks in a computational grid. 


\section{RELATED WORK}

\subsection{Scheduling}

Lan Foster and Car Kesselman (2004) [3] develop a fault tolerant job scheduling strategy in order to tolerate faults gracefully in an economy based grid environment. They propose a novel adaptive task check pointing based fault tolerant job scheduling strategy for an economy based grid. They present a survey with the grid community. The survey reveals that, users have to be highly involved in diagnosing failures, that most failures are due to configuration problems and that solutions for dealing with failures are mainly application-dependent.

\subsection{Heuristic Algorithms}

Heuristic algorithms are used for the static and dynamic tasks assignment problem. Many of these algorithms apply only to the special case where the tasks are independent i.e. with no precedence constraints. Heuristic scheduling algorithms are used in heterogeneous computing environments. These algorithms use historical data of execution time and system load and explicit constraints to schedule jobs.

\subsection{Non-Evolutionary Random Scheduling Algorithm}

Non-evolutionary random scheduling (RS) algorithm is used for efficient matching and scheduling of inter-dependent tasks in a distributed heterogeneous computing (DHC) system. RS is a succession of randomized task orderings and a heuristic mapping from task order to schedule. Randomized task ordering is effectively a topological sort where the outcome may be any possible task order for which the task precedent constraints are maintained.

\subsection{Fault Tolerant Dynamic Scheduling Algorithm}

Manimaran and Murthy (1997) [4] proposed an algorithm for dynamically scheduling arriving real-time tasks with resource and primary-backup-based faulttolerant requirements in a multiprocessor system. This algorithm can tolerate more than one fault at a time and employs techniques such as distance concept, flexible backup overloading and resource reclaiming to improve the guarantee ratio of the system.

They address the problem of building a reliable and highly-available grid service by replicating the service on two or more hosts using the primary-backup approach. The primary goal is to evaluate the ease and efficiency with which this can be done, by first designing a primary-backup protocol using Open Grid Services Infrastructure (OSGI).

\subsection{Primary-Backup Approach}

Primary-backup approach, also called passive replication strategy. In this approach a backup is executed when its primary cannot complete execution due to processor failure. It does not require fault diagnosis and is guaranteed to recover all affected tasks by processor failure. Most works using the primary-backup approach consider scheduling of independent tasks.

\subsubsection{Backup Overloading and Overlapping}

Backup overloading is used to reduce replication cost of independent task which allows scheduling backups of multiple primaries on the same or overlapping time interval on a processor.

In Backup Overlapping, for example, two primary copies are scheduled on processor 1 and processor 3 and their backups are scheduled in an overlapping manner on processor 2 .

\subsection{Backup Schedules}

After the earliest possible start time for a backup on all processor is determined, the time window that this backup can be scheduled on all processor is determined which is between this time and its deadline. Primary schedules and non over loadable backup schedules that are scheduled on the time window can be identified. These backup schedules could be scheduled for independent tasks or dependent tasks as interleaving technique is allowed.

\section{PROPOSED WORK}

The proposed system integrates resource and data source from different geographical environment. In this system, location of resource and data source is identified. There exist a fault-detection mechanism such as fail-signal and acceptance 
test to detect processor and task failures. If a failure is detected in the primary, the backup will execute. Backup resources are designed with replication factors. Impact of communication protocols is considered. Communication protocols are used to distribute the message of each task to grid resource.

\subsection{Scheduling Strategies}

The resources and data source are managed from different environment. The location of resources and data sources is identified. There exist a fault-detection mechanism such as fail-signal and acceptance test to detect processor and task failures. If a failure is detected in the primary, the backup will execute. Backup resources are designed with replication factors. Backup overloading is used for scheduling backups of multiple primaries on the same or overlapping time interval on a processor. Resource reclaiming is also invoked when the primary completes earlier than its estimated execution time. It is necessary so that the backup slot can be released timely for new tasks.

MRC-ECT algorithm is used to schedule the backup of independent job. MCTLRC algorithm is used to schedule the backup of dependent job. For independent tasks, scheduling of backups is independent and backups can overload as long as their primaries are schedule on different processors. Backup scheduling and overloading of dependent tasks are nontrivial and the constraint is that the backup of second task can only start after backup of first task finishes and must not be schedule on the processor where primary of the first task is located.

\subsubsection{MRC-ECT Algorithm}

MRC-ECT algorithm is used for scheduling backup of independent tasks. The objective is to improve resource utilization. For all processor besides the one where the primary is scheduled on, boundary schedules within the time window are considered and their replication cost is compared. This algorithm first considers the left boundary schedules of the time window. It is guaranteed to find an optimal backup schedule in terms of replication cost for a task.

\subsubsection{MCT-LRC Algorithm}

MCT-LRC algorithm is used for scheduling backup of independent tasks. The objective is to reduce job rejection. For all processor besides the one where the primary is scheduled on, boundary schedules within the time window are considered and the boundary schedule which can complete earliest is chosen. This algorithm first considers the left boundary schedules of the time window. Then, all existing schedules within or overlapping with the time window are examined one by one. The algorithm calculates replication cost of the earliest schedule on the current processor and records it.

\subsection{Communication Protocols}

Different communication protocols are used in grid environment. Transmission Control Protocol (FTP) and User Datagram Protocol (UDP) are used for data transmission. Grid File Transfer Protocol (FTP) is used for data transmission. It is used to transfer files in parallel manner. These protocols are used to distribute the message of each task to grid resource. The system analyses the data transmission in task failures.

\section{CONCLUSION}

In this paper, for Grid systems, we addressed the problem of fault- tolerant scheduling of jobs in heterogeneous environment. We considered the impact of communication protocols. Algorithms MRCECT and MCT-LRC for independent and dependent tasks respectively do not require sampling. These algorithms can schedule backups in a much faster way in heterogeneous environment. .

\section{REFERENCES}

[1] Aikebaier.A, Makoto Takizawa, Abawajy. J.H (2004), "Fault-Tolerant Scheduling Policy for Grid Computing Systems" Proceeding on Parallel and Distributed Processing Symposium (IPDPS)..

[2] Al-Omari, R., Somani, A.K., and Manimaran, G. (2001),"A New FaultTolerant Technique for improving Schedulability in Multiprocessor RealTime Systems" Proceedings on Parallel Distributed Processing Symposium (IPDPS). 
[3] Foster .I and Kesselman .C (2004), “Grid: Blueprint for a Future Computing Infrastructure". Morgan Kaufmann

[4] Subbiah A. and Blough D. (2004),'Distributed Diagnosis in Dynamic Fault Environments" Parallel and Distributed Systems.

[5] Qin X. and Jiang H. (2006), "A Novel Fault tolerant Scheduling Algorithm for precedence constrained Tasks in Real-Time Heterogeneous Systems" Parallel Computing.

Author:

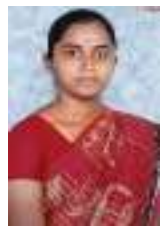

Mrs.R.Kohila received M.E(CSE) degree from Kongu Engineering College(Affiliated to Anna University, Autonomous), Perundurai, India in 2011 and MCA degree from Bannari Amman Institute of Technology (Affiliated to Anna University), Sathyamangalam, India, in 2009 and B.Sc., degree from Trinity College of Arts and Science for women (Affiliated to Periyar University), Namakkal,India, in 2006. She has the experience in Teaching of $3+$ Years. Now she is currently working as an Assistant Professor in V.S.B Engineering College, Karur, Tamil Nadu, and India. His research interests include Data Mining, Advanced Data Bases, Computer Networks etc. She had presented papers in 2 National Conferences so far. 